\title{
Voyager 1 Observations of Galactic Cosmic Rays in the Local Interstellar Medium: Energy Density and Ionization Rates
}

\author{
A. C. Cummings ${ }^{* a}$, E. C. Stone ${ }^{a}$, B. C. Heikkila ${ }^{b}$, N. Lal ${ }^{b}$, W. R. Webber ${ }^{c}$, G. \\ Jóhannesson $^{d}$, I. V. Moskalenko ${ }^{e}$, E. Orlando ${ }^{e}$, and T. A. Porter ${ }^{e}$ \\ ${ }^{a}$ Caltech, Pasadena $C A$ \\ ${ }^{b}$ Goddard Space Flight Center, Greenbelt, $M D$ \\ ${ }^{c}$ New Mexico State University, Las Cruces, NM \\ ${ }^{d}$ University of Iceland, Reykjavik, Iceland \\ e Stanford University, Stanford, CA \\ E-mail: acedsrl.caltech.edu, ecsesrl.caltech.edu, \\ bryant.heikkila@gmail.com, nand. lal@nasa.gov, bwebber@nmsu.edu, \\ gudlaugu@glast2. stanford.edu, imosestanford.edu, \\ eorlandoestanford.edu, tporterestanford.edu
}

\begin{abstract}
Voyager 1 (V1) has been in the local interstellar medium (LISM) since August, 2012. We present the galactic cosmic-ray (GCR) energy spectra of most elements from $\mathrm{H}$ through $\mathrm{Ni}$, and also of electrons, for a period exceeding two years. The V1 energy spectra define the newly-revealed, low-energy part of the interstellar spectra of nuclei down to $\sim 3 \mathrm{MeV} n u c^{-1}$ and of electrons down to $\sim 5 \mathrm{MeV}$. We use a leaky-box model and three GALPROP models of the local interstellar spectra of nuclei, constrained by the V1 observations at low energies and by observations at 1 AU from other missions at higher energies, to estimate the cosmic-ray energy density and the ionization rate of atomic $\mathrm{H}$ in the local interstellar medium by nuclei above $3 \mathrm{MeV}$ nuc $^{-1}$. We use a model of the interstellar electron spectrum that approximately matches the V1 observations to derive the electron contributions to the cosmic-ray energy density and the ionization rate of atomic $\mathrm{H}$ above $3 \mathrm{MeV}$. We find that the total cosmic-ray energy density is in the range 0.82-0.97 $\mathrm{eV} \mathrm{cm}^{-3}$, which includes a contribution of $0.023 \mathrm{eV} \mathrm{cm}^{-3}$ from electrons. We find the cosmicray ionization rate of atomic $\mathrm{H}$ to be in the range $1.45-1.58 \times 10^{-17} \mathrm{~s}^{-1}$, which is a factor of more than 10 below the cosmic-ray ionization rate in diffuse interstellar clouds based on astrochemistry methods. We discuss possible reasons for this difference.
\end{abstract}

The 34th International Cosmic Ray Conference,

30 July- 6 August, 2015

The Hague, The Netherlands

${ }^{*}$ Speaker. 

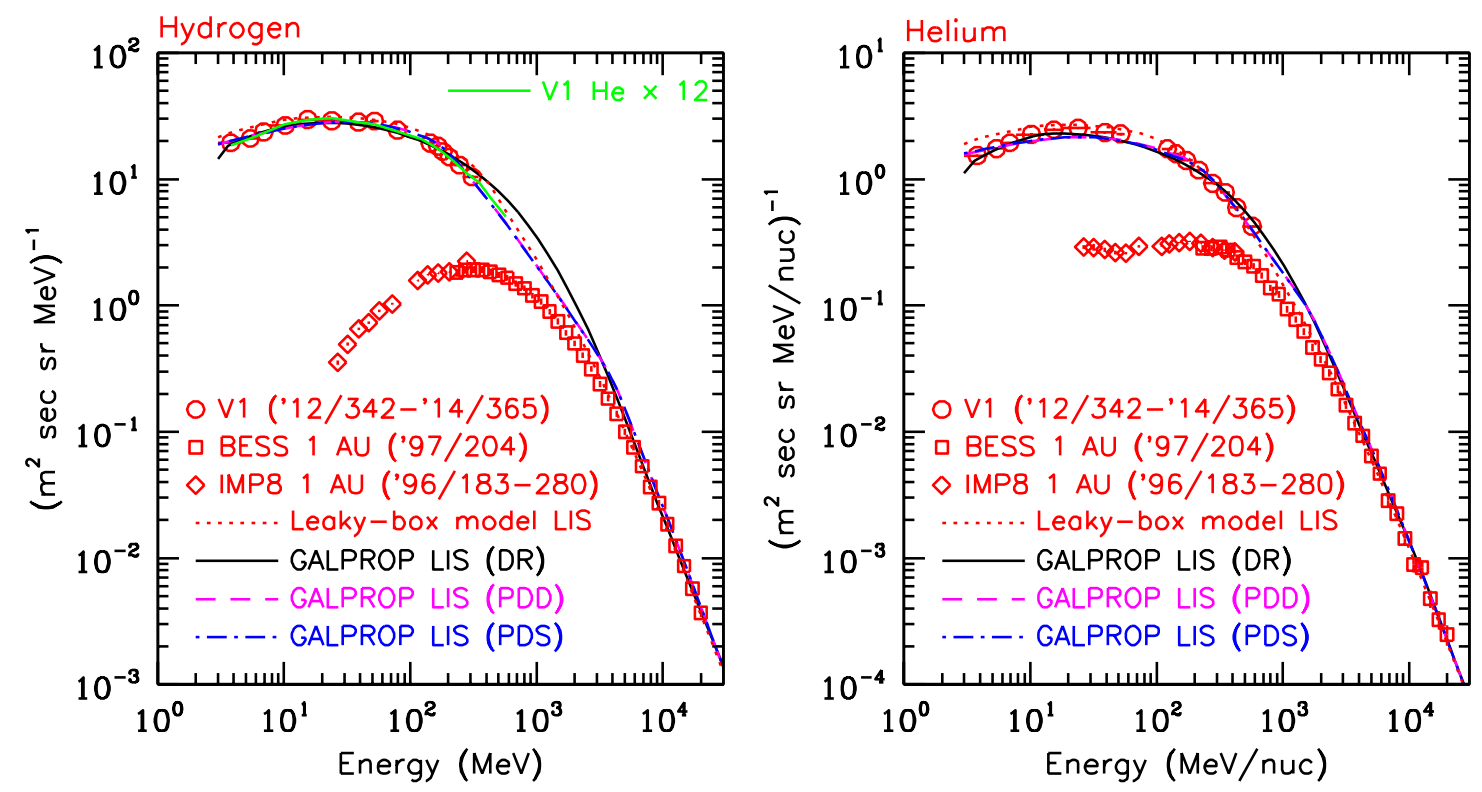

Figure 1: Differential energy spectra of $\mathrm{H}$ (left) and He (right) from V1, and solar-modulated spectra at 1 AU from a BESS balloon flight in 1997 [4], and from IMP8 in the latter part of 1996 [5]. Also shown are estimated spectra in the LISM from a leaky-box model and three GALPROP models as described in the text.

\section{Introduction}

On 25 August 2012, Voyager 1 (V1) entered a region of space in which the heliospheric ions and electrons were depleted and the galactic cosmic ray (GCR) intensity was enhanced [1,2]. It is generally thought that V1 has been in the local interstellar medium (LISM) since that time. We present energy spectra of GCR electrons and most nuclei from $\mathrm{H}$ through $\mathrm{Ni}$ for an $\sim 2$ year period, as well as models of interstellar spectra constrained by these new observations. The models are used to estimate the energy density of cosmic rays in the LISM and the cosmic-ray ionization rate of atomic $\mathrm{H}$ at cosmic-ray energies above $3 \mathrm{MeV} /$ nuc. This work represents an update to a study published in 1998 [3] before there were direct observations of the energy spectra at low energies in the LISM.

\section{Observations, nuclei}

In Figure 1, we show the elemental $\mathrm{H}$ and He spectra from the V1 Cosmic Ray Subsystem (CRS) instrument [6], as well as spectra at $1 \mathrm{AU}$ during a solar minimum time period. The V1 energy spectra of both $\mathrm{H}$ and $\mathrm{He}$ have a broad peak in the $10-50 \mathrm{MeV} \mathrm{nuc}^{-1}$ energy range. Over the energy range of the V1 observations, the GCR H/He ratio in the LISM is $\sim 12$, independent of energy from 3 to $346 \mathrm{MeV} \mathrm{nuc}^{-1}$. The roll over at low energies from a power-law at high energies is likely a consequence primarily of ionization energy losses [7] and suggests that V1 is not in the nearby vicinity of a recent source of GCRs. The estimated interstellar energy spectra from the leaky-box model (LBM) and the three GALPROP models are also shown. The higher-energy portions of the $1 \mathrm{AU}$ spectra are relatively unaffected by solar modulation and constrain the LISM models. 


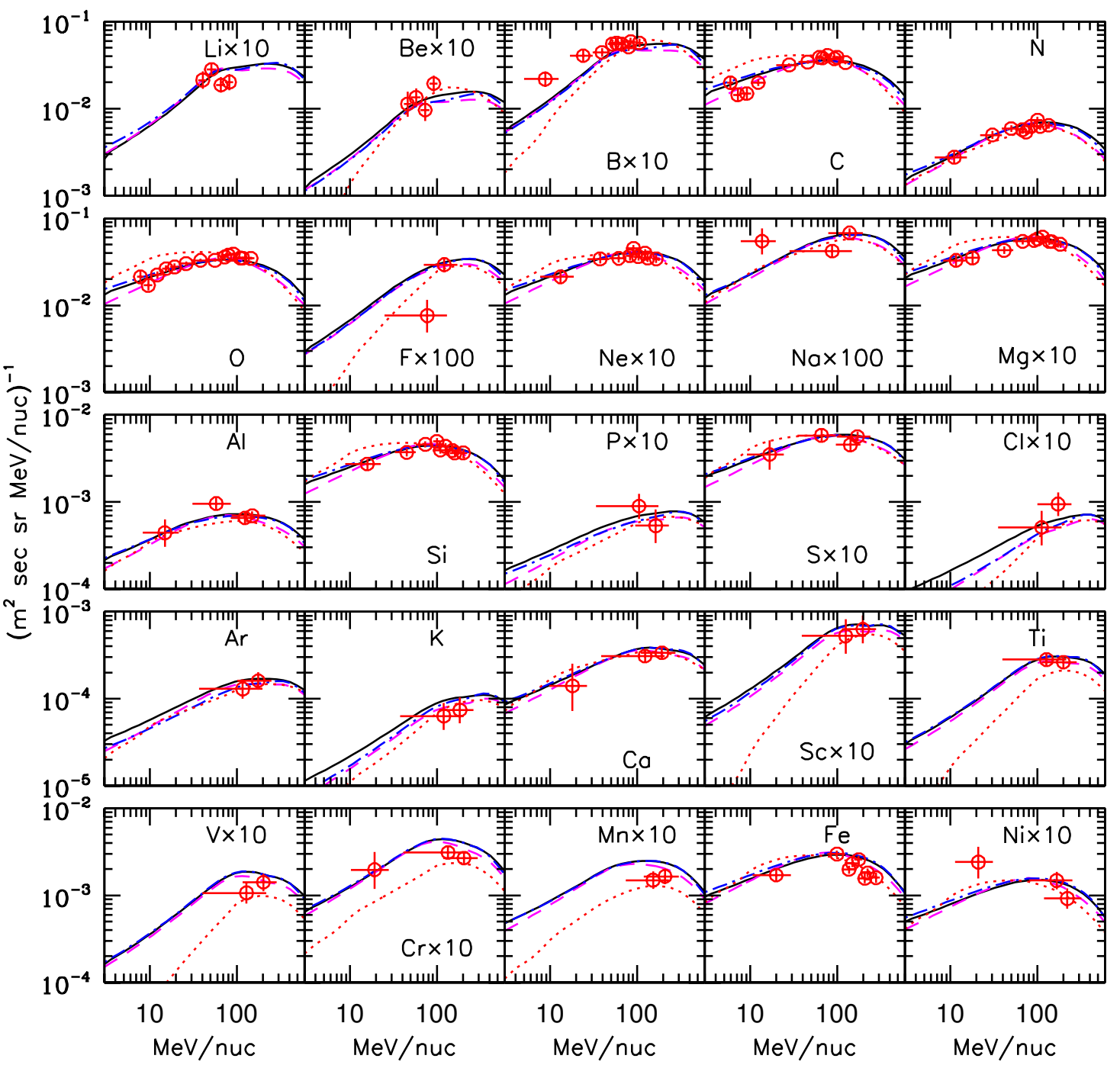

Figure 2: Differential energy spectra from V1. Also shown are estimated spectra in the LISM from a leakybox model and three GALPROP models as described in the text. The line types for the models are the same as in Figure 1.

In Figure 2, we show energy spectra from the CRS instrument on V1 of 25 nuclei from Li through $\mathrm{Ni}$, with the exception of $\mathrm{Co}$, which has yet to be detected. The model spectra in this figure and in Figure 1 are used to calculate the energy density of cosmic rays and the cosmic-ray ionization rate of atomic $\mathrm{H}$ in the LISM.

\section{Models}

The LBM is a version of that used by [12] with updates in the parameters to describe the new data below $\sim 100 \mathrm{MeV} \mathrm{nuc}^{-1}$. The most important parameter is the escape length, $\lambda$, which is taken to be $\lambda=26.5 \beta \mathrm{P}^{-0.5} \mathrm{~g} \mathrm{~cm}^{-2}$ above a rigidity of $1 \mathrm{GV}$ and $\lambda=\mathrm{C} \beta^{1.5}$ below $1 \mathrm{GV}$, where $\mathrm{C}$ $=31.0$ for $\mathrm{H}$ and 40.9 for other nuclei. The source spectra are all assumed to have an exponent $=$ -2.25 . The cross section file has been updated and extended down to $10 \mathrm{MeV} \mathrm{nuc}^{-1}$. 
The GALPROP propagation models have been used in previous studies: diffusive reacceleration (DR) and plain diffusion (PD). The DR model is successful in explaining an ensemble of GCR data [13] and has been used for an extensive study of the Galactic diffuse gamma-ray emission [14]. The propagation parameters of DR and PD models and the elemental source abundances are adjusted to fit the GCR data from V1, ACE CRIS [15], HEAO-3 [16], and CREAM [17]. The PD model, which requires a break in the rigidity dependence of the diffusion coefficient to match the $\mathrm{B} / \mathrm{C}$ ratio, is further modified by introducing a single break in the cosmic-ray injection spectrum in the case of the PDS model, or two breaks in the case of the PDD model. In all models three different cosmic-ray injection spectra are used for protons, $\mathrm{He}$, and heavier elements $\mathrm{Z}>2$.

\section{Observations, electrons}

In Figure 3 we show the energy spectrum of GCR electrons derived from V1 observations, as well as high-energy data from the PAMELA mission at $1 \mathrm{AU}$ [11]. Also shown are several estimates of the interstellar electron spectrum. For the purposes of calculating energy density and ionization rates of atomic $\mathrm{H}$ from GCR

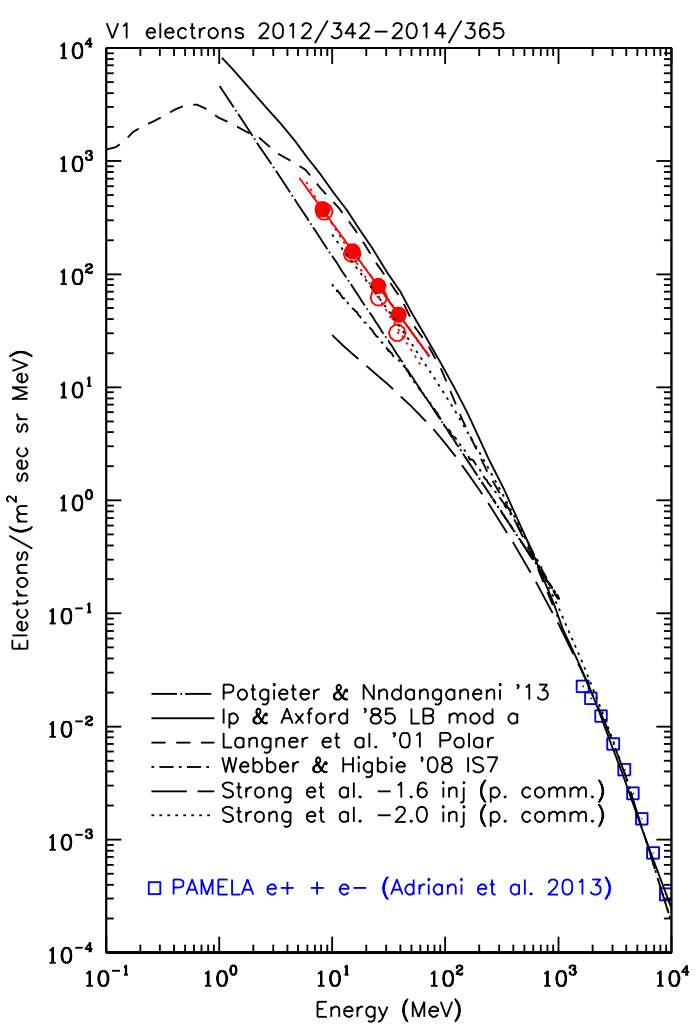

Figure 3: Energy spectrum of electrons (positrons + negatrons) as derived from data from the TET telescope on V1 in two ways, one using response functions measured at an accelerator calibration prior to launch (open symbols) and another one (closed symbols) derived from simulations using the GEANT4 code (www.geant4.cern.ch). Six different estimates of the interstellar energy spectra of electrons are also shown $[8,7,9,10]$. The data at higher energies (open squares) are from the PAMELA mission at $1 \mathrm{AU}[11]$.

electrons, we use the spectrum labeled "Strong et al. -2.0 inj", which was originally derived down to $\sim 0.5 \mathrm{GeV}$ from WMAP observations of the Galactic diffuse synchrotron emission using GALPROP modeling [18]. Subsequently, calculations were extended to $10 \mathrm{MeV}$ (Strong et al., private communication) and finally a further power-law extrapolation with index -1.47 down to 3 $\mathrm{MeV}$ was employed (not shown). Amongst the spectra in Figure 3, this spectrum best matches the V1 observations.

\section{Ionization Rates}

The ionization rate of atomic $\mathrm{H}$ in the LISM by cosmic rays in $\mathrm{s}^{-1}$ is calculated based on 
equation 10 of [19]:

$$
\zeta_{H}=4 \pi \xi_{H} \int_{E_{l o w}}^{E h i g h} J(E) \sigma(E) d E
$$

where $\xi_{H}=1.5$ to account for ionization due to secondary electrons produced in the initial ionizing event [20] and $\mathrm{E}_{\text {low }}$ is $3 \mathrm{MeV}$ for GCR protons and electrons and $3 \mathrm{MeV} \mathrm{nuc}^{-1}$ for GCR nuclei. The values of $\mathrm{E}_{\text {high }}$ were $1.59 \times 10^{6} \mathrm{MeV}$ for electrons, $1.07 \times 10^{9} \mathrm{MeV} \mathrm{nuc}^{-1}$ for the GALPROP models, and $1.0 \times 10^{7} \mathrm{MeV}$ nuc $^{-1}$ for the leaky-box model. $\mathrm{J}(\mathrm{E})$ is the differential energy spectrum of the GCR ionizing species and $\sigma(\mathrm{E})$ is the energy-dependent cross section.

The cross section used is the Bethe-Bloch formulation [21], valid at the relatively high energies used here. The equation for $\sigma$ in $\mathrm{cm}^{2}$ as presented in [22] is:

$$
\sigma=1.23 \times 10^{-20} Z^{2}\left[6.2+\log _{10}\left(\beta^{2} /\left(1-\beta^{2}\right)-0.43 \beta^{2}\right] / \beta^{2}\right.
$$

where $\mathrm{Z}$ is the charge of the ionizing particle and $\beta \mathrm{c}$ is the particle velocity.

At energies significantly lower than the V1 measurements, the Bethe-Bloch formula overestimates the cross section. In Figure 4 we show the cross section for ionizing atomic $\mathrm{H}$ by GCR protons and He nuclei with energies $10^{-5} \mathrm{MeV} \mathrm{nuc}^{-1}$ to $10^{6} \mathrm{MeV} \mathrm{nuc}^{-1}$, where for values of $\beta<0.026$ we show the cross section formulation adapted for ionization of $\mathrm{H}$ from [23] given in $\mathrm{cm}^{2}$ for an incident ion of charge $\mathrm{Z}$ and velocity $\mathrm{v}$ in $\mathrm{cm} / \mathrm{s}$ :

$$
\sigma=4 \pi a_{o}^{2} Z^{2} G\left[v /\left(2.19 \times 10^{8} \sqrt{(} Z+1\right)\right]
$$

where $a_{o}=5.29 \times 10^{-9}$ and the function $\mathrm{G}$ is given by:

$$
G(x)=\exp \left(-1 / x^{2}\right)\left[1.26+0.283 \ln \left(2 x^{2}+25\right)\right] / x^{2}
$$

Also shown in Figure 4 is the cross section for ionizing atomic $\mathrm{H}$ by GCR electrons, where for electron energies $\mathrm{E}<1000 \mathrm{eV}$ we use the cross section formulation of $[24,25]$ as presented in [26]:

$$
\sigma=4 \times 10^{-14}[1-0.6 \exp -0.56(E / 13.6-1)] \ln (E / 13.6) /(13.6 E)
$$

\section{Energy Density}

The equation for calculating energy density is adapted from [19] and is given by $\epsilon_{C R}=$ $4 \pi N \int_{E_{l o w}}^{E_{\text {high }}} E(J(E) / v) d E$, where $\mathrm{v}$ is the particle velocity in $\mathrm{cm} \mathrm{s}^{-1}$ and the intensity $\mathrm{J}(\mathrm{E})$ is in $\left(\mathrm{cm}^{2} \mathrm{~s} \mathrm{sr} \mathrm{eV}\right)^{-1}$ for protons and electrons, where $\mathrm{N}=1$, and in $\left(\mathrm{cm}^{2} \mathrm{~s} \mathrm{sr} \mathrm{eV} \mathrm{nuc}-1\right)^{-1}$ for heavier ions with $\mathrm{N}$ being the number of nucleons. The limits of integration are as in Equation 5.1, except $\mathrm{eV}$ and $\mathrm{eV} \mathrm{nuc}^{-1}$ are the units used instead of $\mathrm{MeV}$ and $\mathrm{MeV}_{\mathrm{nuc}}{ }^{-1}$.

\section{Results and Summary}


Table 1: Energy density and ionization rate of atomic $\mathrm{H}$.

\begin{tabular}{crrrrrrrrrr} 
Nuclei Model & \multicolumn{4}{c}{ Energy density $\left(\mathrm{eV} \mathrm{cm}^{-3}\right)$} & \multicolumn{4}{c}{ Ionization rate $\left(10^{-17} \mathrm{~s}^{-1}\right)$} \\
& $\mathrm{H}$ & $\mathrm{He}$ & $\mathrm{Li}-\mathrm{Ni}$ & $\mathrm{e}$ & Total & $\mathrm{H}$ & $\mathrm{He}$ & $\mathrm{Li}-\mathrm{Ni}$ & $\mathrm{e}$ & Total \\
LBM & 0.58 & 0.15 & 0.07 & 0.023 & 0.82 & 0.85 & 0.27 & 0.29 & 0.17 & 1.58 \\
DR & 0.70 & 0.17 & 0.08 & 0.023 & 0.97 & 0.80 & 0.24 & 0.31 & 0.17 & 1.51 \\
PDD & 0.63 & 0.17 & 0.07 & 0.023 & 0.89 & 0.77 & 0.24 & 0.28 & 0.17 & 1.45 \\
PDS & 0.62 & 0.17 & 0.07 & 0.023 & 0.89 & 0.76 & 0.24 & 0.30 & 0.17 & 1.47
\end{tabular}

The resulting ionization rates and energy densities are shown in Table 1. $\mathrm{H}$ and $\mathrm{He}$ dominate the contributions in all cases. The electron contribution is the same for each nuclei model entry. The total cosmic-ray ionization rate varies from $1.45 \times 10^{-17} \mathrm{~s}^{-1}$ to $1.58 \times 10^{-17} \mathrm{~s}^{-1}$, which is a factor of 11-12 lower than the cosmicray ionization rate of $1.78 \times 10^{-16}$ $\mathrm{s}^{-1}$ inferred using astrochemistry methods for diffuse interstellar clouds [27]. As seen in Figure 4, however, the cross section for ionization peaks at energies much

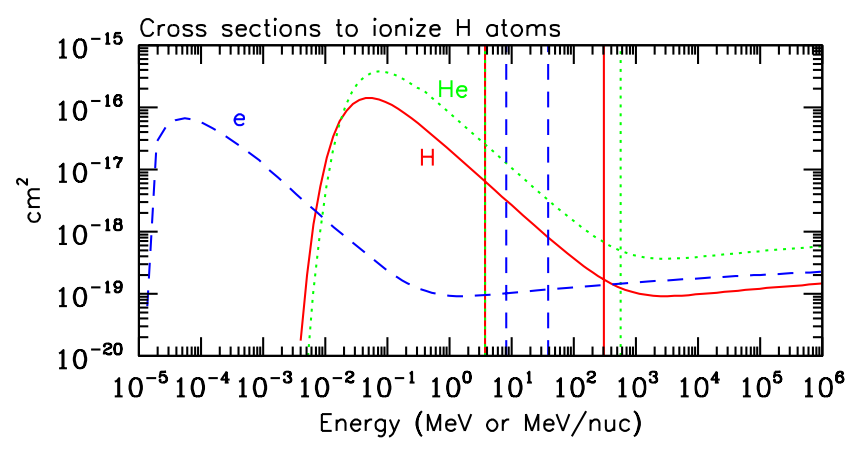

Figure 4: Cross section in $\mathrm{cm}^{2}$ for ionization of atomic $\mathrm{H}$ by energetic electrons, $\mathrm{H}$ ions, and $\mathrm{He}$ ions. The curves are from equations 5.2, 5.3, 5.4, and 5.5. The vertical lines represent the energy range of the V1 observations with solid being for $\mathrm{H}$, dotted for $\mathrm{He}$, and dashed for electrons.

lower than the V1 observations. Most attempts to resolve the discrepancy in the literature involve allowing the then unknown interstellar energy spectra of GCRs protons, for example, to turn up below energies of $\sim 100 \mathrm{MeV}[19,28]$. However, the new V1 observations suggest that there is no turn up at energies above $3 \mathrm{MeV}$.

Among the possibilities to explain the difference are a spatially variable GCR energy spectrum in the Galaxy, especially the larger ionization rate is expected in spiral arms which host GCR sources and thus higher densities of GCRs, a contribution from knock-on electrons below $\sim 1 \mathrm{MeV}$, and possibly a contribution from a suprathermal tail on the energy distribution of the interstellar wind. We note that suprathermal tails are ubiquitous in the heliosphere and are often attributed to a pump mechanism [29]. We find that, for example, a suprathermal tail on the Maxwell-Boltzmann energy distribution of the interstellar wind of $\mathrm{H}$ ions with an energy spectrum of $4.4 \times 10^{-3} \mathrm{E}^{-1.5}$ $\exp [-\mathrm{E} /(0.2 \mathrm{MeV})]\left(\mathrm{cm}^{2} \mathrm{~s} \mathrm{sr} \mathrm{MeV}\right)^{-1}$ would result in approximately the increase required in the ionization rate of atomic $\mathrm{H}$ with negligible change in the energy density. It remains for future work to see if the interstellar turbulence is large enough to sustain such a suprathermal tail and to explore the other possibilities mentioned above.

Whereas the ionization rate is a relatively low-energy phenomenon, energy density is a relatively high-energy phenomenon with most of the contribution coming at energies above the 
V1 measurement energy range (see Figure 4 of [19]). We find that the total energy density amongst the models ranges from 0.82 to $0.97 \mathrm{eV} \mathrm{cm}^{-3}$.

The interstellar magnetic field would have an intensity of 5.7-6.2 $\mu \mathrm{G}$ if there were equipartition. Although the observed intensity in the very local interstellar medium is nearly this large, ranging from $\sim 4$ to $5.5 \mu \mathrm{G}$, this is likely enhanced due to draping around the heliosphere, and the unperturbed field is expected to be in the range of 2 to $4 \mu \mathrm{G}$ [30].

The cosmic-ray ionization rate we derive is lower by a factor of $\sim 2$ than that derived by Webber [3] $\left(3-4 \times 10^{-17} \mathrm{~s}^{-1}\right)$. The energy density we derive is also lower than in [3] $(\sim 1.8 \mathrm{eV}$ $\mathrm{cm}^{-3}$ ). We ascribe the differences to the different interstellar energy spectra used, with the new V1 GCR electron measurements reducing the electron contributions to the ionization rate significantly.

\section{Acknowledgments}

Voyager data analysis is supported by NASA Grant NNN12AA012. GALPROP development is supported by NASA Grants NNX13AC47G and NNX10AE78G.

\section{References}

[1] E. C. Stone et al., Voyager 1 Observes Low-Energy Galactic Cosmic Rays in a Region Depleted of Heliospheric Ions, Science 341 (July, 2013) 150-153.

[2] S. M. Krimigis et al., Search for the Exit: Voyager 1 at Heliosphere's Border with the Galaxy, Science 341 (July, 2013) 144-147.

[3] W. R. Webber, A New Estimate of the Local Interstellar Energy Density and Ionization Rate of Galactic Cosmic Cosmic Rays, Astrophys. J. 506 (Oct., 1998) 329-334.

[4] Y. Shikaze et al., Measurements of 0.2-20 GeV/n cosmic-ray proton and helium spectra from 1997 through 2002 with the BESS spectrometer, Astroparticle Physics 28 (Sept., 2007) 154-167, [astro-ph/0611388].

[5] F. B. McDonald, Cosmic-Ray Modulation in the Heliosphere A Phenomenological Study, Space Sci. Rev. 83 (Jan., 1998) 33-50.

[6] E. C. Stone et al., Cosmic Ray Investigation for the Voyager Missions: Energetic Particle Studies in the Outer Heliosphere - and Beyond, Space Sci. Rev. 21 (1977) 355-376.

[7] W.-H. Ip and W. I. Axford, Estimates of galactic cosmic ray spectra at low energies, Astron. Astrophys. 149 (Aug., 1985) 7-10.

[8] M. S. Potgieter and R. R. Nndanganeni, A local interstellar spectrum for galactic electrons, Astroparticle Physics 48 (Aug., 2013) 25-29, [arXiv: 1302 .2424].

[9] U. W. Langner et al., Proposed local interstellar spectra for cosmic ray electrons, International Cosmic Ray Conference 10 (Aug., 2001) 3992.

[10] W. R. Webber and P. R. Higbie, Limits on the interstellar cosmic ray electron spectrum below $\sim 1-2$ $\mathrm{GeV}$ derived from the galactic polar radio spectrum and constrained by new Voyager 1 measurements, J. Geophys. Res. (Space Physics) 113 (Nov., 2008) 11106.

[11] O. Adriani et al., Cosmic-Ray Positron Energy Spectrum Measured by PAMELA, Physical Review Letters 111 (Aug., 2013) 081102, [arXiv: 1308.0133]. 
[12] W. R. Webber and P. R. Higbie, Galactic propagation of cosmic ray nuclei in a model with an increasing diffusion coefficient at low rigidities: A comparison of the new interstellar spectra with Voyager data in the outer heliosphere, J. of Geophys. Res. (Space Physics) 114 (Feb., 2009) 2103.

[13] R. Trotta et al., Constraints on Cosmic-ray Propagation Models from A Global Bayesian Analysis, Astrophys. J. 729 (Mar., 2011) 106, [arXiv: 1011.0037$].$

[14] M. Ackermann et al., Fermi-LAT Observations of the Diffuse $\gamma$-Ray Emission: Implications for Cosmic Rays and the Interstellar Medium, Astrophys. J. 750 (May, 2012) 3, [arXiv: 1202.4039 ].

[15] J. S. George et al., Elemental Composition and Energy Spectra of Galactic Cosmic Rays During Solar Cycle 23, Astrophys. J. 698 (June, 2009) 1666-1681.

[16] J. J. Engelmann et al., Charge composition and energy spectra of cosmic-ray nuclei for elements from Be to NI - Results from HEAO-3-C2, Astron. Astrophys. 233 (July, 1990) 96-111.

[17] Y. S. Yoon et al., Cosmic-ray Proton and Helium Spectra from the First CREAM Flight, Astrophys. J. 728 (Feb., 2011) 122, [arXiv:1102.2575].

[18] A. W. Strong et al., The interstellar cosmic-ray electron spectrum from synchrotron radiation and direct measurements, Astron. Astrophys. 534 (Oct., 2011) A54, [arXiv:1108.4822].

[19] N. Indriolo et al., The Implications of a High Cosmic-Ray Ionization Rate in Diffuse Interstellar Clouds, Astrophys. J. 694 (Mar., 2009) 257-267, [arXiv : 0901.1143 ].

[20] A. E. Glassgold and W. D. Langer, Model calculations for diffuse molecular clouds, Astrophys. J. 193 (Oct., 1974) 73-91.

[21] H. Bethe, Quantenmechanik Der Ein- und Zwei-Elektronenprobleme, Band 1, in Handbuch der Physik, pp. 24, 273-560. Springer, Berlin, 1933.

[22] L. Spitzer, Jr. and M. G. Tomasko, Heating of H I Regions by Energetic Particles, Astrophys. J. 152 (June, 1968) 971.

[23] I. D. Kaganovich et al., Scaling cross sections for ion-atom impact ionization, Physics of Plasmas 11 (Mar., 2004) 1229-1232.

[24] W. Lotz, Electron-Impact Ionization Cross-Sections and Ionization Rate Coefficients for Atoms and Ions, Astrophys. J. Supp. 14 (May, 1967) 207.

[25] W. Lotz, An empirical formula for the electron-impact ionization cross-section, Zeitschrift fur Physik 206 (Apr., 1967) 205-211.

[26] M. Bzowski et al., Solar Parameters for Modeling the Interplanetary Background, p. 67. 2013.

[27] N. Indriolo et al., Herschel Survey of Galactic $\mathrm{OH}^{+}, \mathrm{H}_{2} \mathrm{O}^{+}$, and $\mathrm{H}_{3} \mathrm{O}^{+}$: Probing the Molecular Hydrogen Fraction and Cosmic-Ray Ionization Rate, Astrophys. J. 800 (Feb., 2015) 40, [arXiv:1412.1106].

[28] M. E. Wiedenbeck, Cosmic-Ray Energy Spectra and Time Variations in the Local Interstellar Medium: Constraints and Uncertainties, Space Sci. Rev. 176 (June, 2013) 35-46.

[29] L. A. Fisk and G. Gloeckler, Particle Acceleration in the Heliosphere: Implications for Astrophysics, Space Sci. Rev. 173 (Nov., 2012) 433-458.

[30] L. F. Burlaga et al., In Situ Observations of Magnetic Turbulence in the Local Interstellar Medium, Astrophys. J. Lett. 804 (May, 2015) L31. 